\title{
Estimation of copy number aberrations: Comparison of exome sequencing data with SNP microarrays identifies homozygous deletions of 19q13.2 and $C I C$ in neuroblastoma
}

\author{
SUSANNE FRANSSON $^{1}$, MALIN ÖSTENSSON ${ }^{1}$, ANNA DJOS $^{1}$, NILOUFAR JAVANMARDI ${ }^{1}$, \\ PER KOGNER $^{2}$ and TOMMY MARTINSSON ${ }^{1}$
}

${ }^{1}$ Department of Medical and Clinical Genetics, Sahlgrenska Academy at University of Gothenburg, Gothenburg, Sweden;
${ }^{2}$ Childhood Cancer Research Unit, Department of Women's and Children's Health, Karolinska Institutet, Stockholm, Sweden

Received October 29, 2015; Accepted December 8, 2015

DOI: $10.3892 /$ ijo.2016.3349

\begin{abstract}
In the pediatric cancer neuroblastoma, analysis of recurrent chromosomal aberrations such as loss of chromosome 1p, 11q, gain of $17 \mathrm{q}$ and $M Y C N$ amplification are used for patient stratification and subsequent therapy decision making. Different analysis techniques have been used for detection of segmental abnormalities, including fluorescence in situ hybridization (FISH), comparative genomic hybridization (CGH)-microarrays and multiplex ligation-dependent probe amplification (MLPA). However, as next-generation sequencing becomes available for clinical use, this technique could also be used for assessment of copy number alterations simultaneously with mutational analysis. In this study we compare genomic profiles generated through exome sequencing data with profiles generated from high resolution Affymetrix single nucleotide polymorphism (SNP) microarrays on 30 neuroblastoma tumors of different stages. Normalized coverage reads for tumors were calculated using Control-FREEC software and visualized through a web based Shiny application, prior to comparison with corresponding SNP-microarray data. The two methods show high-level agreement for breakpoints and copy number of larger segmental aberrations and numerical aneuploidies. However, several smaller gene containing deletions that could not readily be detected through the SNP-microarray analyses were identified through exome profiling, most likely due to difference between spatial distribution of microarray probes and targeted regions of the exome capture. These smaller aberrations included focal ATRX deletion in two tumors and three cases of novel deletions in chromosomal region 19q13.2 causing homozygous loss of multiple genes including the $C I C$ (Capicua) gene. In conclusion, genomic profiles
\end{abstract}

Correspondence to: Dr Susanne Fransson, Department of Medical and Clinical Genetics, University of Gothenburg, P.O. Box 445, SE-405 30 Gothenburg, Sweden

E-mail: susanne.fransson@clingen.gu.se

Key words: neuroblastoma, pediatric cancer, genomic alterations, Capicua generated from normalized coverage of exome sequencing show concordance with SNP microarray generated genomic profiles. Exome sequencing is therefore a useful diagnostic tool for copy number variant (CNV) detection in neuroblastoma tumors, especially considering the combination with mutational screening. This enables detection of theranostic targets such as $A L K$ and $A T R X$ together with detection of significant segmental aneuploidies, such as 2p-gain, 17q-gain, 11q-deletion as well as $M Y C N$ amplification.

\section{Introduction}

The childhood cancer neuroblastoma (NB) is a tumor of the sympathetic nervous system. The patients show high degree of clinical and biological heterogeneity ranging from patients with highly aggressive tumors with fatal outcome, even after intense multimodal treatment, to patients with spontaneous regression despite metastatic disease. Tumors with whole chromosome gains or losses and near triploid karyotypes generally have a good prognosis while tumors with segmental rearrangements and near di- or tetraploid karyotype are associated with a poor prognosis. Consequently, analyses of chromosomal aberrations are an important tool, that together with age at diagnosis, presence of metastases, tumor differentiation and histological grade are used for patient stratification and to determine therapeutic strategy (1). Recurrent genomic alterations with clinical importance include loss of chromosome 1p, 3p, 4p, 11q, gain of 1q, 2p, 17q and amplification of the MYCN oncogene where 11q-deletion and MYCNamplification both are strongly associated with aggressive disease (2-4). Different analysis techniques commonly used in clinical routine for detection of segmental and numerical aberrations include karyotyping, fluorescence in situ hybridization (FISH), comparative genomic hybridization (CGH) microarrays or multiplex ligation-dependent probe amplification (MLPA) $(5,6)$. However, cancer diagnostics is moving towards a paradigm with tests that perform comprehensive characterization of genomic alterations of individual tumors such as next generation sequencing (NGS) in order to detect actionable targets. Depending on settings, NGS could provide information of different sorts of therapeutically relevant 
alterations such as single nucleotide variants, indels and translocations. In addition, by analysis of the distribution of read-depth between test tumor sample and normalization control, it is possible to identify gains, losses, amplifications as well as homozygous deletions (7-10). Thus, NGS provides an attractive tool in clinical diagnostics of neuroblastoma, enabling simultaneous assessment of copy number alterations alongside detection of point mutations (e.g., activating mutations in the $A L K$ oncogene) that could be used to aid in choice of therapy. In order to investigate the accuracy of genomic alterations characterized from exome sequencing, exome data for 30 neuroblastoma tumors were compared with corresponding genomic profiles generated from established Affymetrix high resolution SNP-microarrays, the golden standard for copy number detection in neuroblastoma in many laboratories.

\section{Materials and methods}

Samples and microarray analysis. The collection of tumors from Swedish patients were performed after either written or verbal consent was obtained from parents/guardians according to ethical permits approved by the local ethics committee (Karolinska Institutet and Karolinska University Hospital, registration number 03-736 and 2009/1369). Clinical information is described in Table I. The tumors were histologically assessed for tumor cell content using adjacent tissue and genomic DNA was isolated from fresh frozen tumor or blood using DNeasy blood and tissue kit (Qiagen, Hilden, Germany) according to the manufacturer's protocol. DNA concentration and purity were assessed through fluorometric analysis and absorbance measurements, respectively.

Microarray analysis of 30 neuroblastoma tumors were performed using either Affymetrix $50 \mathrm{~K}, 250 \mathrm{~K}$ gene mapping arrays or CytoScan HD (Affymetrix Inc., Santa Clara, CA, USA) containing 59,015, 262,338 and 2,822,125 probes respectively (corresponding to $\sim 50,10$ and $1 \mathrm{~kb}$ average probe spacing). Handling of the microarrays has been described previously $(2,11)$. Primary data analysis was performed using GDAS software (Affymetrix) with in silico normalization against control samples from healthy individuals. Genomic position annotations were based on the hg19 build (http:// genome.ucsc.edu/) of the human genome.

Exome sequencing and $C N V$-analysis. Exome sequencing was performed on DNA from 30 neuroblastoma tumors and corresponding constitutional blood from 14 of these patients using Agilent SureSelect All Exon 50Mb V3 or V4 (Agilent, Santa Clara, CA, USA) according to the suppliers protocol before performing pair-end sequencing (2x100 bp or 2x75 bp) on Illumina HiSeq2000 or HiScan SQ (Illumina, San Diego, CA, USA). The sequencing was performed at three separate occasions at SciLife laboratory, Stockholm, Sweden and the Genomics Core facility at University of Gothenburg, Sweden with a median raw coverage of $91 \mathrm{X}, 127 \mathrm{X}$ and $340 \mathrm{X}$ for each batch (Table I). Reads were aligned against the reference genome (hg19) using BWA-0.5.10 after fastq trimming with prinseq 0.20 .3 prior realignment and recalibration using GATK-2.5-.-gf57256b. Variant calling were made through SNPeff followed by Annovar annotation.
Copy number alterations were generated from the bioinformatical tool Control-FREEC (control-FREE Copy Number Caller) (10) using the normalized distribution of aligned reads in window-by-window basis, in order to determine differences in coverage between tumor and normal. Neuroblastoma tumors were compared against either constitutional DNA from blood from corresponding patient or from that of other controls. The ratios generated with Control-FREEC were visualized using the statistical software $\mathrm{R}$. We constructed a web-based Shiny application (12) that runs with $\mathrm{R}$ in the background. The Shiny application for visualizing ControlFREEC profiles is available at https://malinost.shinyapps.io/ CNPupload, and the source code at https:/gist.github.com/ malinost/324f77309eb103147747. The application imports the ratios from an uploaded Control-FREEC output file, and visualizes the results either chromosome by chromosome, or for the whole genome in one figure. In the single chromosome mode the values can also be colored according to e.g., copy number or genotype. In order to detect segmental changes and breakpoints we need to extract segmental averages and detect the jumps that correspond to gains or losses, this is done by applying the Fused Lasso Signal Approximator (FLSA) (13) to the ratios. With different settings we can adjust how much the variations in the ratios affect the FLSA lines. In the whole genome setting ratios are plotted with different colors for different chromosomes in proportion to chromosome size. Results of CNV detection from exome sequencing were compared to SNP-microarray through visual annotation in order to determine the performance of respective platform.

\section{Results}

Ratio profiles of tumor-control read coverage were generated using Control-FREEC and visualized with the Shiny application. Break points for structural rearrangements were recorded through FREEC and the plotted profiles were compared to profiles generated from SNP microarrays. Visual annotation shows that the two different methods have high degree of concordance regarding larger alterations (Fig. 1). Both platforms detected a significant number of imbalances including gains, amplification, deletion and homozygous deletions, as well as whole chromosome gains and losses in the thirty analyzed tumor samples. The genomic positions recorded for copy number changes shows close consistency between exome generated profiles and SNP-microarray profiles (Fig. 1), commonly separated with $<1 \mathrm{Mb}$. Among the four deviations recorded when comparing copy number variation generated from SNP-microarray and exome sequencing analysis, two were located in the ATRX gene where sample NBL28R8 and NBL10R8 showed loss of exon 3-8 and exon 1-15, respectively and one were located at chromosome 19: 42.75-44.93 Mb from p-terminal end (pter) in sample NBL13E6 (Table II). However, probe density in these regions is low in the $50 \mathrm{k}$ and $250 \mathrm{~K}$ arrays and thus, the smaller deletions detected through coverage read plots are below the resolution of the arrays used in this study. One additional deviation between the two methods was seen for sample NBL10R8 where copy number profiling through exome and SNP-microarray analysis both indicates 2 p-gain. Intriguingly, the two methods show highly different $2 \mathrm{p}$ gain breakpoint; exome analysis show gain of the entire chromo- 
Table I. Clinical data and sequencing settings.

\begin{tabular}{|c|c|c|c|c|c|c|}
\hline \multicolumn{4}{|c|}{ Clinical information } & \multicolumn{3}{|c|}{ Sequencing information } \\
\hline PatID & INSS & INRG & Outcome & Enrichment kit & Read length & Raw coverage \\
\hline NBL12R6 & 4 & M & DOD & $50 \mathrm{Mb} \mathrm{V4}$ & $2 \times 100$ & 379 \\
\hline NBL28R2 & 3 & $\mathrm{~L}$ & NED & $50 \mathrm{Mb} \mathrm{V4}$ & $2 \times 100$ & 242 \\
\hline NBL49R0 & 4 & M & AWD & $50 \mathrm{Mb} \mathrm{V4}$ & $2 \times 100$ & 321 \\
\hline NBL9R9 & 3 & M & DOD & $50 \mathrm{Mb} \mathrm{V} 3$ & $2 \times 75$ & 136 \\
\hline NBL10R8 & 3 & $\mathrm{~L}$ & DOD & $50 \mathrm{Mb} \mathrm{V} 3$ & $2 \times 75$ & 128 \\
\hline NBL46R6 & 4 & M & NA & $50 \mathrm{Mb} \mathrm{V} 3$ & $2 \times 75$ & 129 \\
\hline NBL15R8 & 3 & $\mathrm{~L}$ & DOD & $50 \mathrm{Mb} \mathrm{V} 3$ & $2 \times 75$ & 102 \\
\hline NBL11E1 & 4 & M & NED & $50 \mathrm{Mb} \mathrm{V} 3$ & $2 \times 100$ & 117 \\
\hline NBL30R0 & 4 & M & NED & $50 \mathrm{Mb} \mathrm{V} 3$ & $2 \times 100$ & 135 \\
\hline NBL13R1 & 3 & $\mathrm{~L}$ & DOD & $50 \mathrm{Mb} \mathrm{V} 3$ & $2 \times 100$ & 90 \\
\hline NBL11E8 & 1 & $\mathrm{~L}$ & NED & $50 \mathrm{Mb} \mathrm{V} 3$ & $2 \times 100$ & 62 \\
\hline NBL44R5 & 1 & $\mathrm{~L}$ & NED & $50 \mathrm{Mb} \mathrm{V} 3$ & $2 \times 100$ & 100 \\
\hline NBL12E3 & $4 \mathrm{~S}$ & M & DOD & $50 \mathrm{Mb} \mathrm{V4}$ & $2 \times 100$ & 364 \\
\hline NBL13E5 & 3 & $\mathrm{~L}$ & DOD & $50 \mathrm{Mb} \mathrm{V4}$ & $2 \times 100$ & 338 \\
\hline NBL13E6 & 3 & $\mathrm{~L}$ & DOD & $50 \mathrm{Mb} \mathrm{V4}$ & $2 \times 100$ & 340 \\
\hline NBL18E2 & 1 & $\mathrm{~L}$ & NED & $50 \mathrm{Mb} \mathrm{V} 3$ & $2 \times 75$ & 119 \\
\hline NBL3E2 & 4 & M & DOD & $50 \mathrm{Mb} \mathrm{V} 3$ & $2 \times 75$ & 120 \\
\hline NBL4E1 & 4 & M & DOD & $50 \mathrm{Mb} \mathrm{V} 3$ & $2 \times 75$ & 127 \\
\hline NBL6E9 & 3 & $\mathrm{~L}$ & DOD & $50 \mathrm{Mb} \mathrm{V} 3$ & $2 \times 75$ & 156 \\
\hline NBL11E4 & 4 & M & DOD & $50 \mathrm{Mb} \mathrm{V} 3$ & $2 \times 75$ & 141 \\
\hline NBL14R2 & $4 \mathrm{~S}$ & M & DOD & $50 \mathrm{Mb}$ V4 & $2 \times 100$ & 122 \\
\hline NBL19R6 & 1 & $\mathrm{~L}$ & DOD & $50 \mathrm{Mb} \mathrm{V4}$ & $2 \times 100$ & 291 \\
\hline NBL25R6 & 4 & M & DOD & $50 \mathrm{Mb} \mathrm{V} 3$ & $2 \times 100$ & 99.8 \\
\hline NBL28R8 & 4 & M & DOD & $50 \mathrm{Mb} \mathrm{V} 3$ & $2 \times 75$ & 132 \\
\hline NBL39R1 & 4 & M & NED & $50 \mathrm{Mb} \mathrm{V4}$ & $2 \times 100$ & 321 \\
\hline NBL46R2 & 4 & M & AWD & $50 \mathrm{Mb} \mathrm{V4}$ & $2 \times 100$ & 362 \\
\hline NBL47R4 & 4 & M & DOD & $50 \mathrm{Mb} \mathrm{V} 3$ & $2 \times 75$ & 126 \\
\hline NBL49R1 & 4 & M & AWD & $50 \mathrm{Mb} \mathrm{V} 3$ & $2 \times 75$ & 101 \\
\hline NBL50R1 & 4 & M & DOD & $50 \mathrm{Mb} \mathrm{V4}$ & $2 \times 100$ & 376 \\
\hline NBL56R2 & 4 & M & AWD & $50 \mathrm{Mb} \mathrm{V4}$ & $2 \times 100$ & 240 \\
\hline
\end{tabular}

INSS, International Neuroblastoma Staging System; INRG, International Neuroblastoma Risk Group; NED, no evidence of disease; DOD, dead of disease; AWD, alive with disease; NA, information not available.

some $2 \mathrm{p}$ arm (92.2 $\mathrm{Mb}$ from pter) while microarrays show a gain ending at $48.2 \mathrm{Mb}$ from pter (Fig. 2).

Additional aberrations of specific interest included one tumor showing chromothriptic features of chromosome 2, 5 and 7 causing amplification of MYCN, TERT and CDK6 and three tumors showing loss of $C D K N 2 A$ (Fig. 1).

Through the copy number analysis of exome sequencing we observed that three tumors (NBL4E1, NBL6E9 and NBL13E6) carried smaller deletions causing homozygous loss in chromosomal region 19q13.2. The shortest region of overlap (SRO) of deletions are delimitated by sample NBL13E6 showing loss of chr19:42,749,732-42,931,020 containing the genes ERF, CIC, PAFAH1B2, PRR19, TMEM145, MEGF8, CNFN, LIPE-AS1 and LIPE (Fig. 3 and Table I). A minor heterozygous deletion was observed in sample NBL12E3 also at 19q13.2 albeit distally (43.34-43.53 Mb from pter) relative the three samples with homozygous loss at $19 \mathrm{q}$. Of the genes located within the region delimitated by sample NBL16E9, rare variants (e.g. not present in 1,000 genome database, exome variant server or our in house database) were detected in MEGF8 (p.P195L, NBL13E5) and in LIPE (p.G627S, NBL47R4; p.A351V, NBL19R6). All three variants were predicted by SIFT to be non-deleterious.

Tumor specific alterations, such as rearrangements could be used to monitoring residual disease and treatment response. Although MYCN amplification is common in neuroblastoma, 
Numerical only profile - NBL11E8

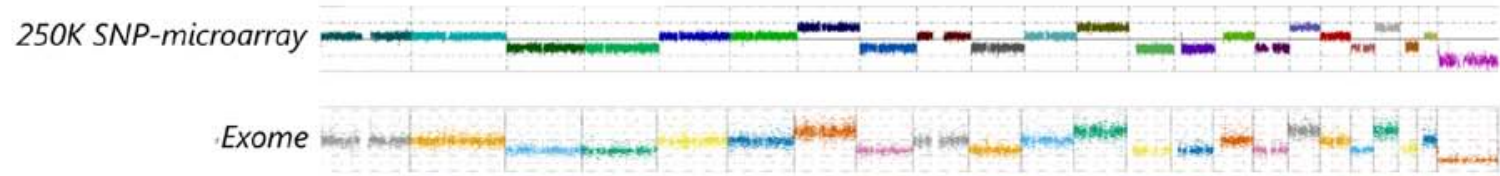

MNA profile - NBL56R2

CytoScan HD SNP-microarray

Exome

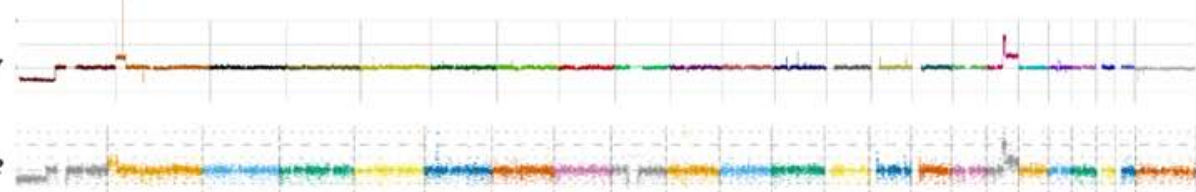

MNA profile - NBL39R1

250K SNP-microarray _- -

Exome

11q profile - NBL47R4

$250 K$ SNP-microarray $\longrightarrow$

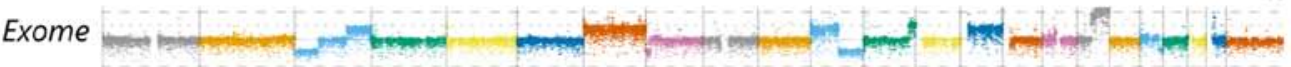

11q profile - NBL4E1

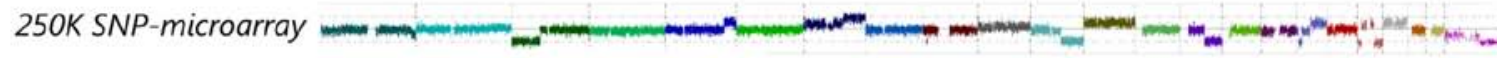

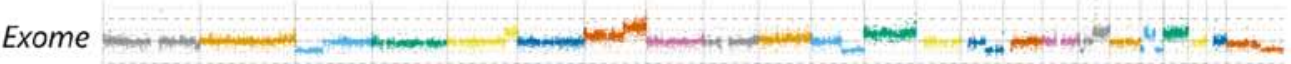

11q profile - NBL10R8

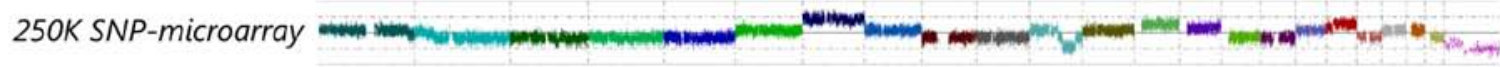

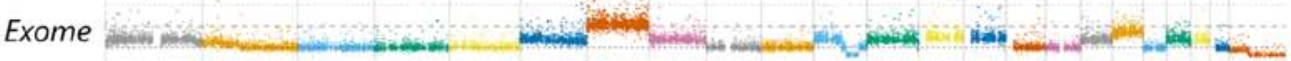

\section{Chromothriptic/11q profile - NBL3E2}

50K SNP-microarray mote then

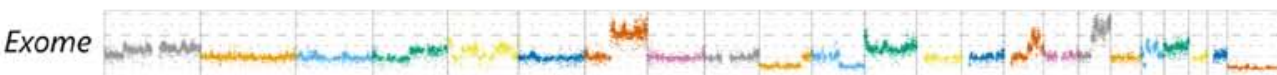

\section{Chromothriptic/MNA profile - NBL49R0}

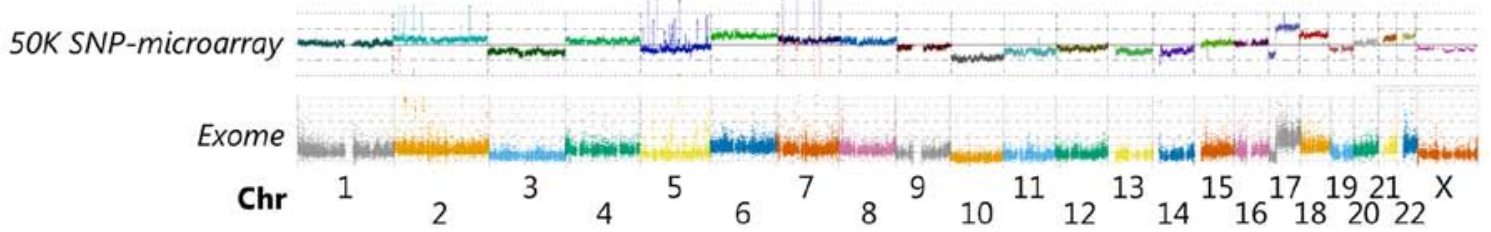

Figure 1. Side-by-side comparison of SNP microarray and exome sequencing generated copy number profiles. Genome-wide copy number profiles are shown for eight neuroblastoma tumors with different genomic characteristic associated with different NB subgroups (2), e.g., numerical only, $M Y C N$ amplification (MNA), 11q-deleted (11q) or chromothriptic features. Upper panel shows chromatogram generated from SNP microarrays and lower panel shows normalized exome coverage plot for the respective sample.

the exact start and end-points of amplicons are unique for each patient and thus requires precise characterization of each tumor. Exome sequencing shows that neuroblastoma samples NBL14R2, NBL13E5 and NBL19R6 display complex amplicon 


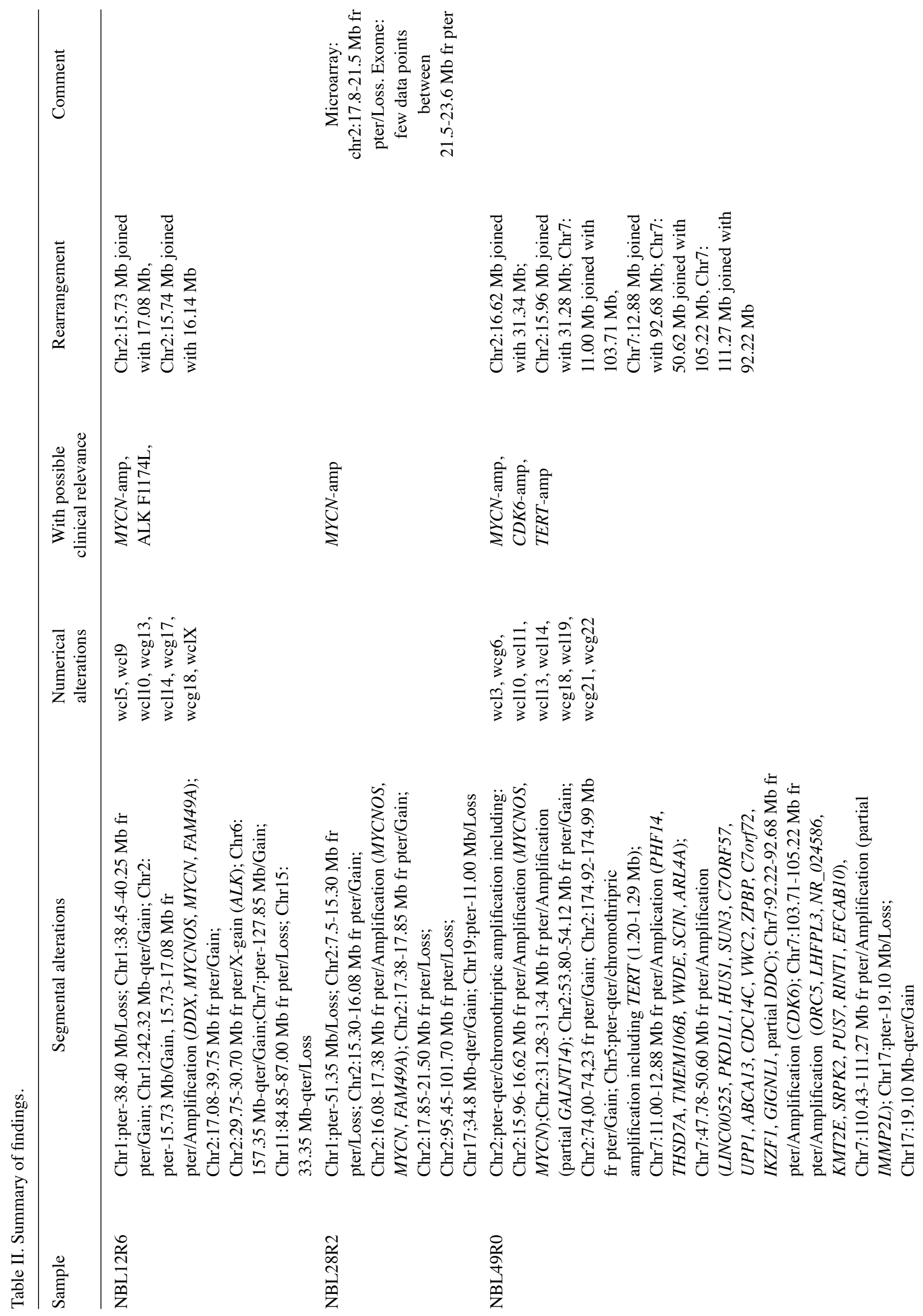




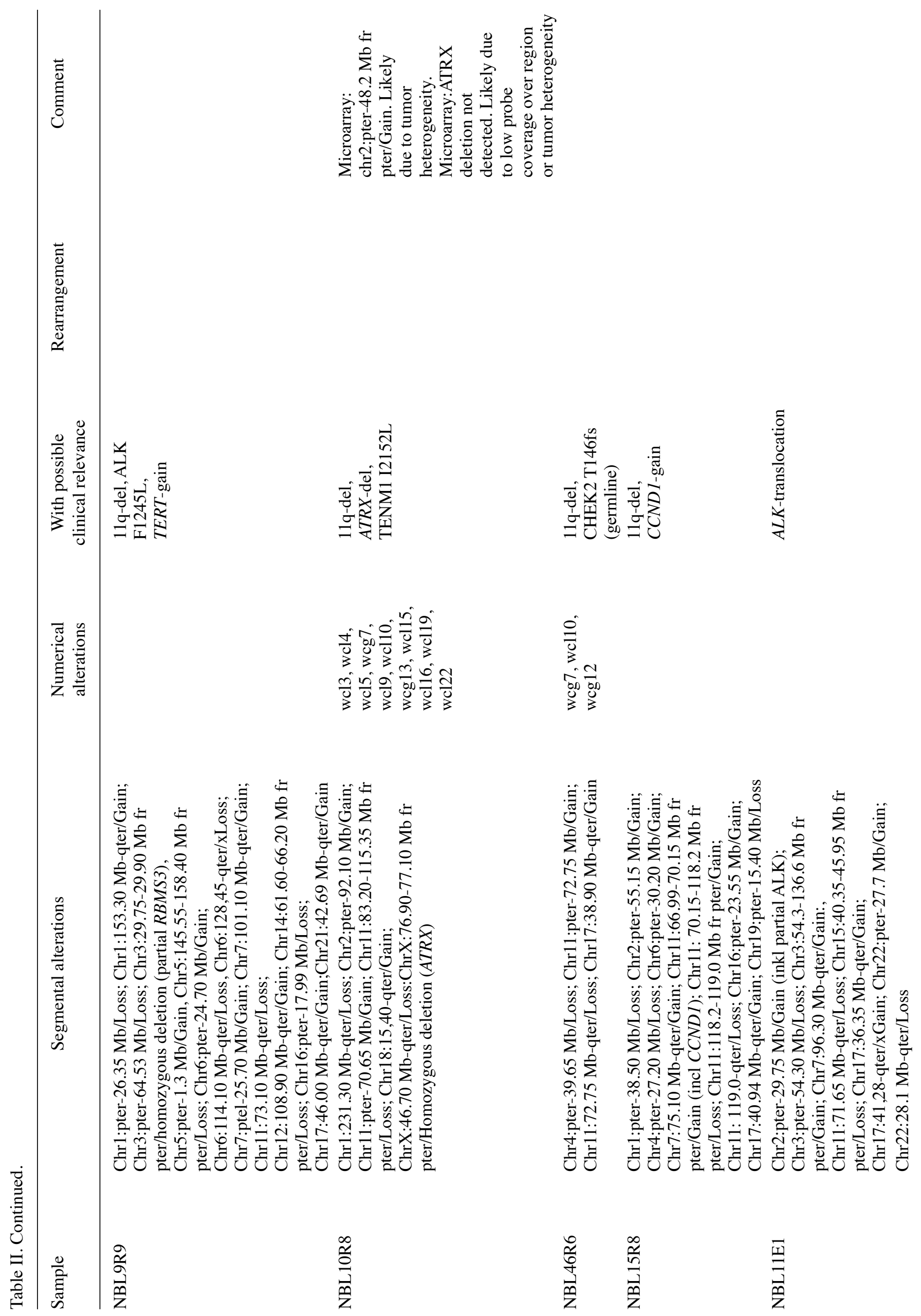




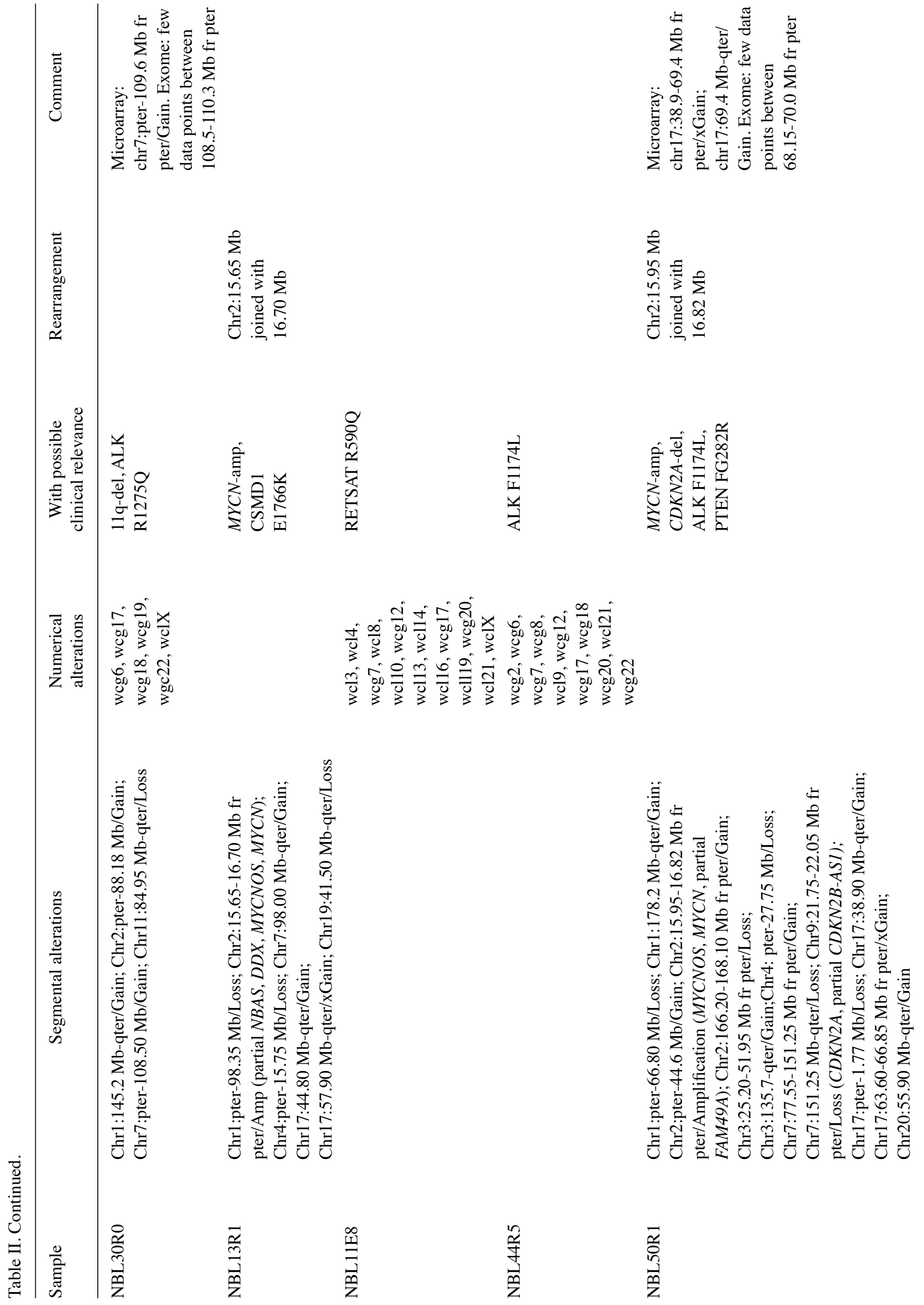




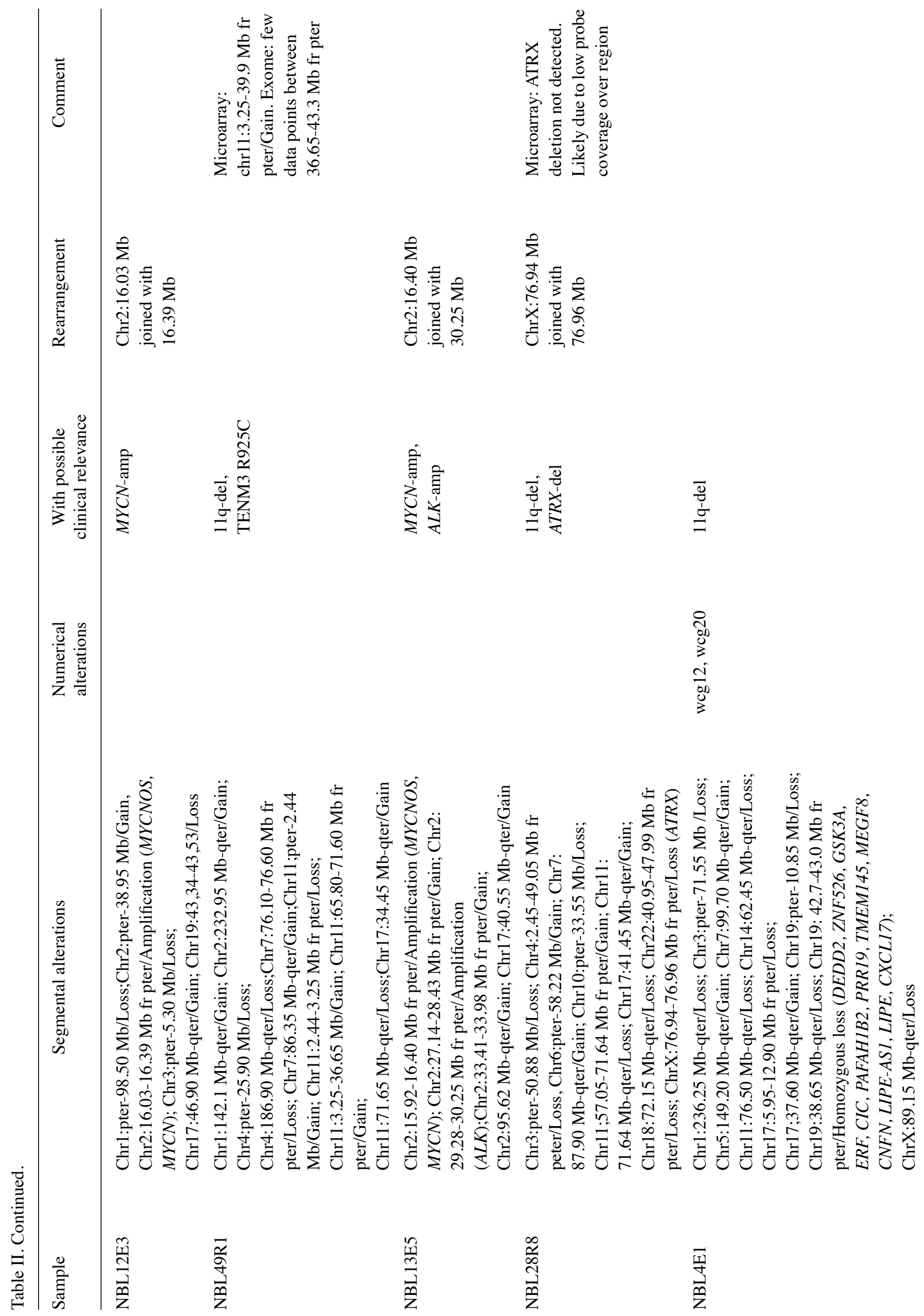




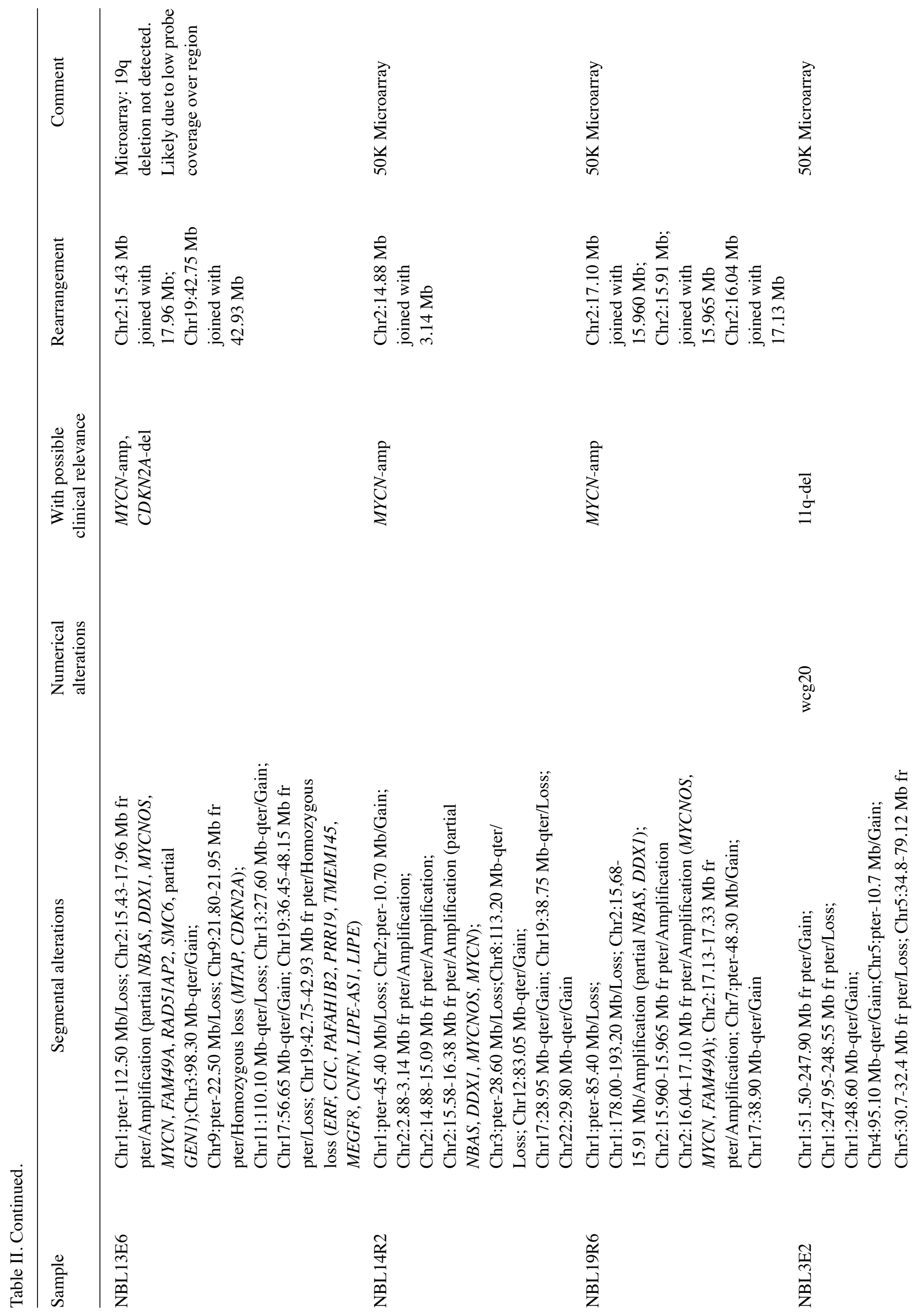




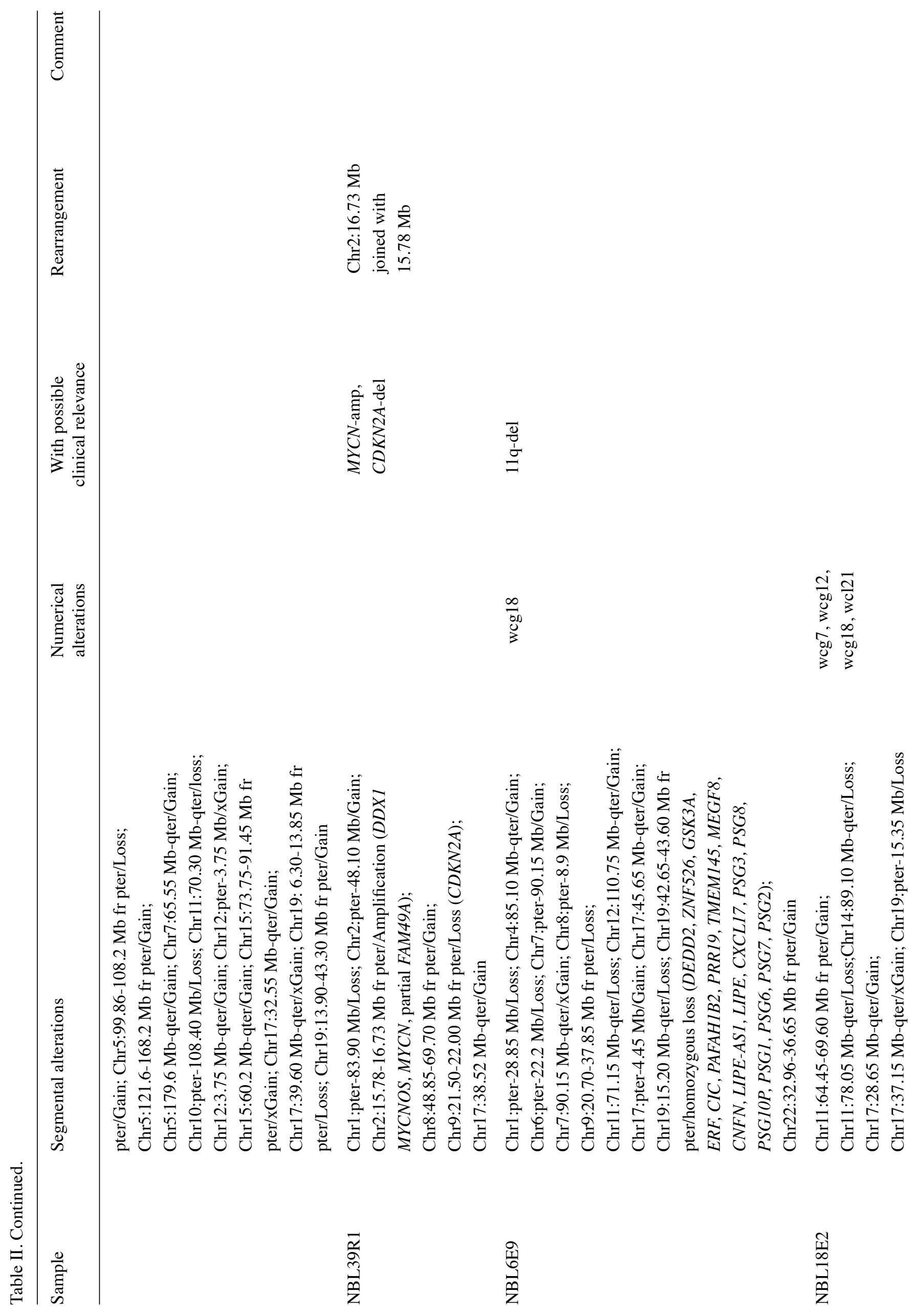




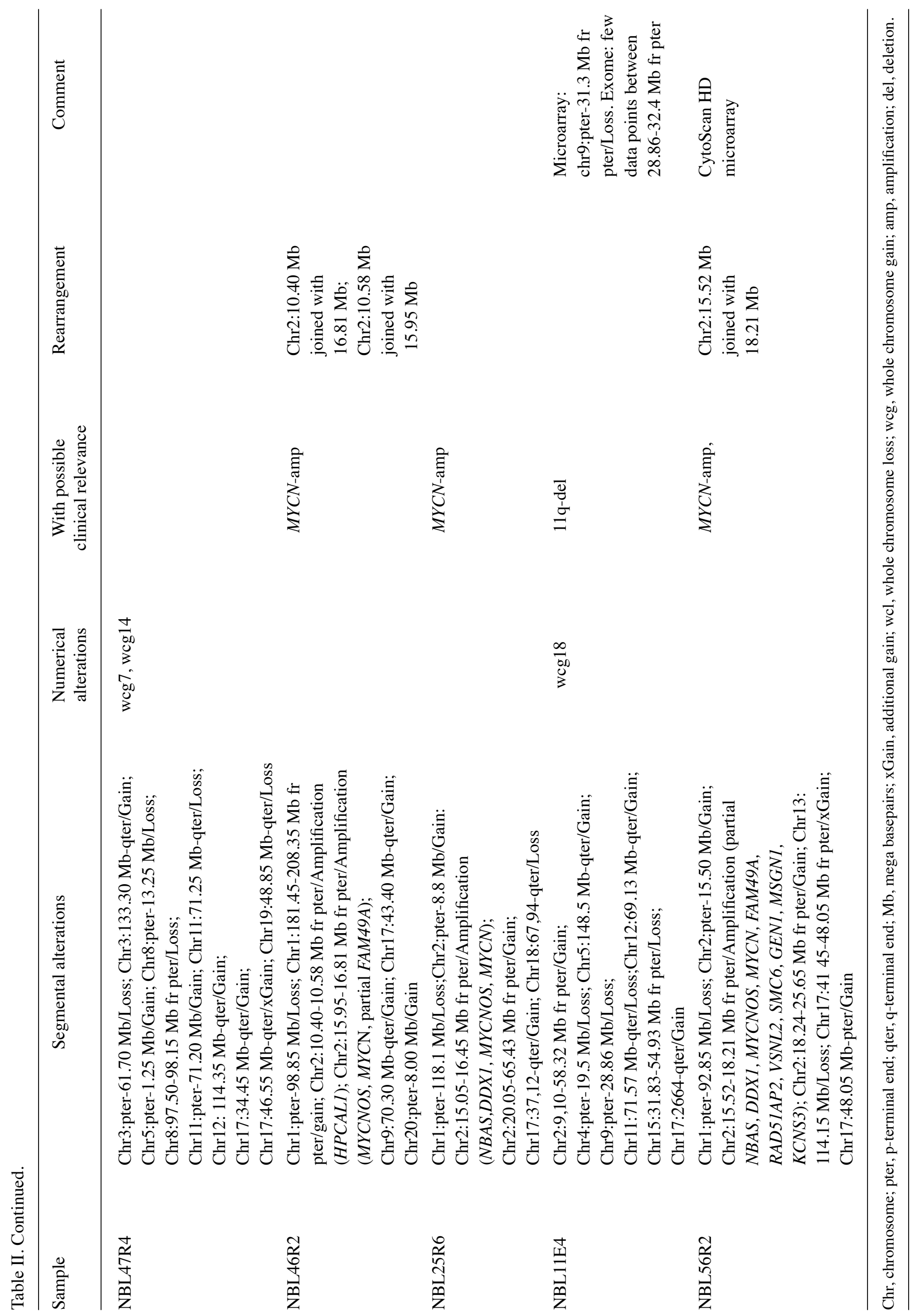


Chr2 - NBL10R8

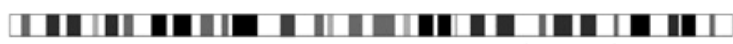

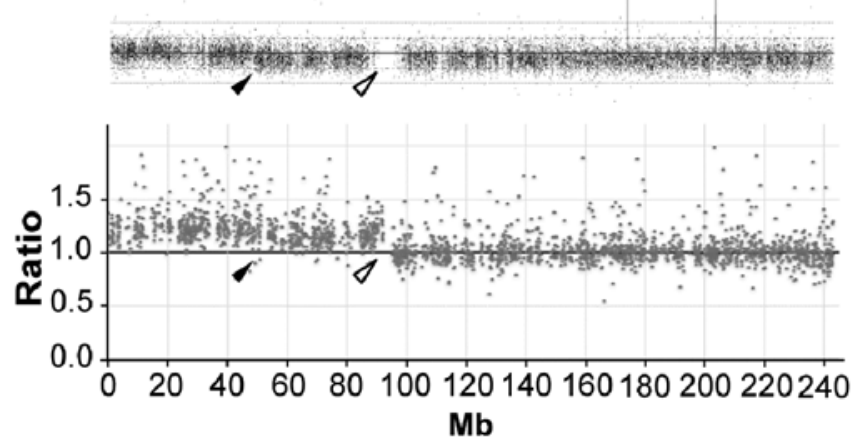

Figure 2. Difference in break point for $2 \mathrm{p}$ gain between the two methods. Gain of chromosomal region $2 \mathrm{p}$ is indicated through both SNP microarray (upper panel) and exome sequencing (lower panel) albeit at different positions. Black arrows show position of break at $48.2 \mathrm{Mb}$ from pter and hollow arrows show position of break at $92.2 \mathrm{Mb}$ from pter identified through SNP microarray and exome profiling, respectively.

structures consisting of multiple $2 \mathrm{p}$-regions in addition to the genomic region containing the $M Y C N$ gene; NBL14R2: 2.88-3.14 Mb, 14.88-15.09 $\mathrm{Mb}$ and 15.58-16.38 $\mathrm{Mb}$ from pter; NBL13E5: 15.92-16.40 Mb and 29.28-30.25 Mb from pter; NBL19R6: 15.68-15.91 Mb, 15.960-15.965 Mb, 16.0417.10 $\mathrm{Mb}$ and $17.13-17.33 \mathrm{Mb}$ from pter. The breakpoints provide several tumor and patient specific junction sites useful for analyses of minimal residual disease follow-up. The highlevel amplification seen in $M Y C N$-amplified tumors produces a great amount of off-target reads within the amplified regions that can be used to deduct specific breakpoints and fusions. From the exome sequencing data we were able to retrieve at least one unique junction site for 12 of 14 NB tumors $(86 \%)$ with $M Y C N$-amplification. We could also identify unique junction sites due to deletions in two patients: NBL28R8 with a deletion in $A T R X$ located at the X-chromosome where 76.94 $\mathrm{Mb}$ joined 76.96 $\mathrm{Mb}$ and NBL13E6 with a small interstitial chromosome 19 deletion fusing position $42.75 \mathrm{Mb}$ with 42.93 Mb.

\section{Discussion}

Next generation sequencing has recently entered clinical practice in evaluation of cancer development and progression. This ranges from use of targeted cancer gene panels to whole genome sequencing. In neuroblastoma there are few recurrent mutations in well-established cancer genes besides $A L K$ (14-17) and thus, other approaches beyond cancer gene panels is required for exploring the mutational landscape. Although sequencing at the whole genome level is reaching affordable levels for clinical utility, the bioinformatic handling and data storage is still a bottleneck for many laboratories. Thus, exome sequencing provides an attractive approach for identification of protein changing mutations for theranostic purposes in neuroblastoma. In this context it is also important to note that the cost of exome sequencing has dropped dramatically in the last five years.

The ability to use exome data for detection of segmental and whole chromosome alterations is crucial as the genomic profile of a neuroblastoma tumor is highly important for decision of treatment regime and indicates prognosis for the patient. In order to analyze the utility of exome based copy number profiling in neuroblastoma we performed a visual annotation of genomic profile and compared exome and SNP-microarray generated profiles of thirty neuroblastoma samples.

The Shiny application for visualization of normalized coverage ratios, developed by us and presented here, allows for a rapid overview of the genomic profiles. Side-by-side comparison of profiles generated from the two different methods show high degree of concordance indicating that exome generated copy number profiles could be used for clinical interpretation. Using exome sequence data we readily detected high level amplifications as well as hetero- and homozygous deletions of gene containing regions (Figs. 1 and 3). Through the exome sequencing data we were able to detect smaller deletions of gene containing genomic regions that were not recorded in the SNP-microarray generated profiles due to inadequate probe density, thereby showing the power of the exome sequencing. However, it is likely that the opposite would be seen also for imbalances occurring outside the targeted regions. When analyzing SNP-microarray profiles an estimation of the minimal number of probes is needed to take into account in order to avoid false positives, and similar considerations should also be addressed in exome generated profiles e.g., by using a sliding window approach. Control-FREEC apply a sliding window approach in several steps, first raw CNP is calculated by counting reads in non-overlapping windows. After the raw CNPs are normalized against a normal sample, a Lasso-based segmentation-algorithm is applied (18).

Besides the resolution of smaller deletions in gene containing regions, only one major deviation was seen in comparing the two methods; in sample NBL10R8 gain of chromosomal region $2 \mathrm{p}$ is detected through both methods although with different end-points (Fig. 2). Tracing the source of DNA indicated that DNA extraction was performed on separate occasions and that the difference in breakpoints at $2 p$ likely is due to tumor heterogeneity.

Interestingly, we were able to detect three cases of homozygous deletions at $19 \mathrm{q}$ causing loss of multiple genes including the CIC gene (Fig. 3). This gene encodes the HMG-box transcriptional repressor Capicua. Capicua is a key sensor of multiple receptor tyrosine kinases (RTK), repressing RTK-responsive genes in absence of activating signaling and is involved in various biological processes including neuroblast differentiation (19). CIC mutations, deletions or truncating mutations are seen in the majority of oligodendrogliomas (20) and Capicua has also been implicated in other malignancies such as breast-, colorectal and prostate cancer $(21,22)$. Allelic loss of the $19 \mathrm{q}$ region and recurrent mutations of $C I C$ is a common feature of oligodendroglioma and frequently seen in combination with allelic loss of $1 \mathrm{p}$ and/or deletions of the far upstream element (FUSE) binding protein 1 gene $F U B P 1$ on $1 \mathrm{p} 31.1$ or mutations of the isocitrate dehydrogenase genes (IDH1/2) (20). However, allelic loss of FUBPI is only seen in one of the samples with homozygous 19q deletion (NBL13E6) and no novel protein changing variant could be detected in $I D H 1 / 2, F U B P 1$ or $C I C$ in our set of 30 neuroblastoma tumors. If and how $C I C$ deficiency contributes to cancer progression in neuroblastoma tumors requires further studies. A hetero- 
A

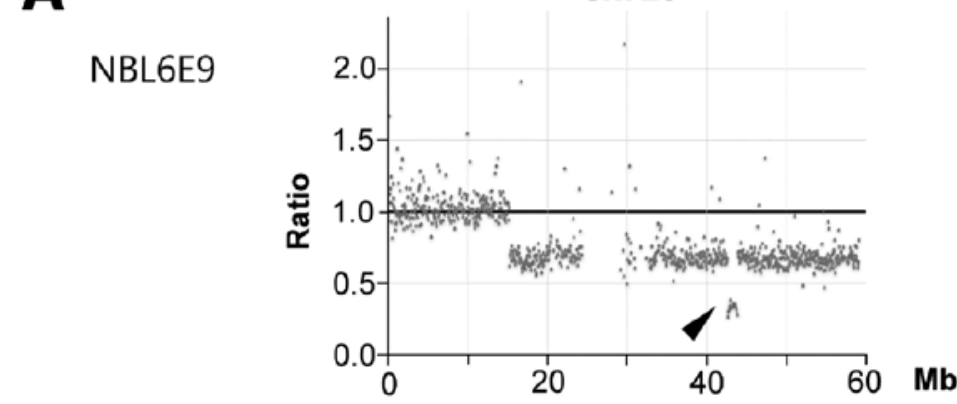

NBL4E1

NBL13E6

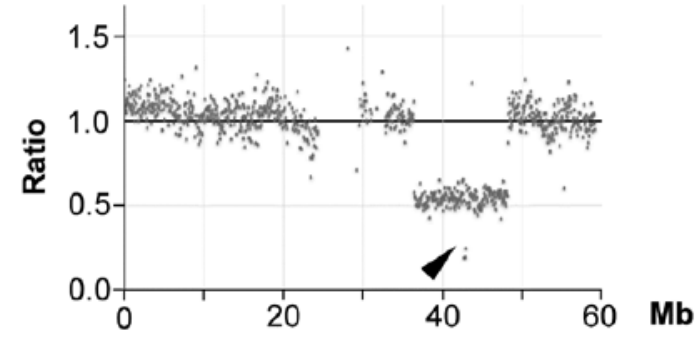

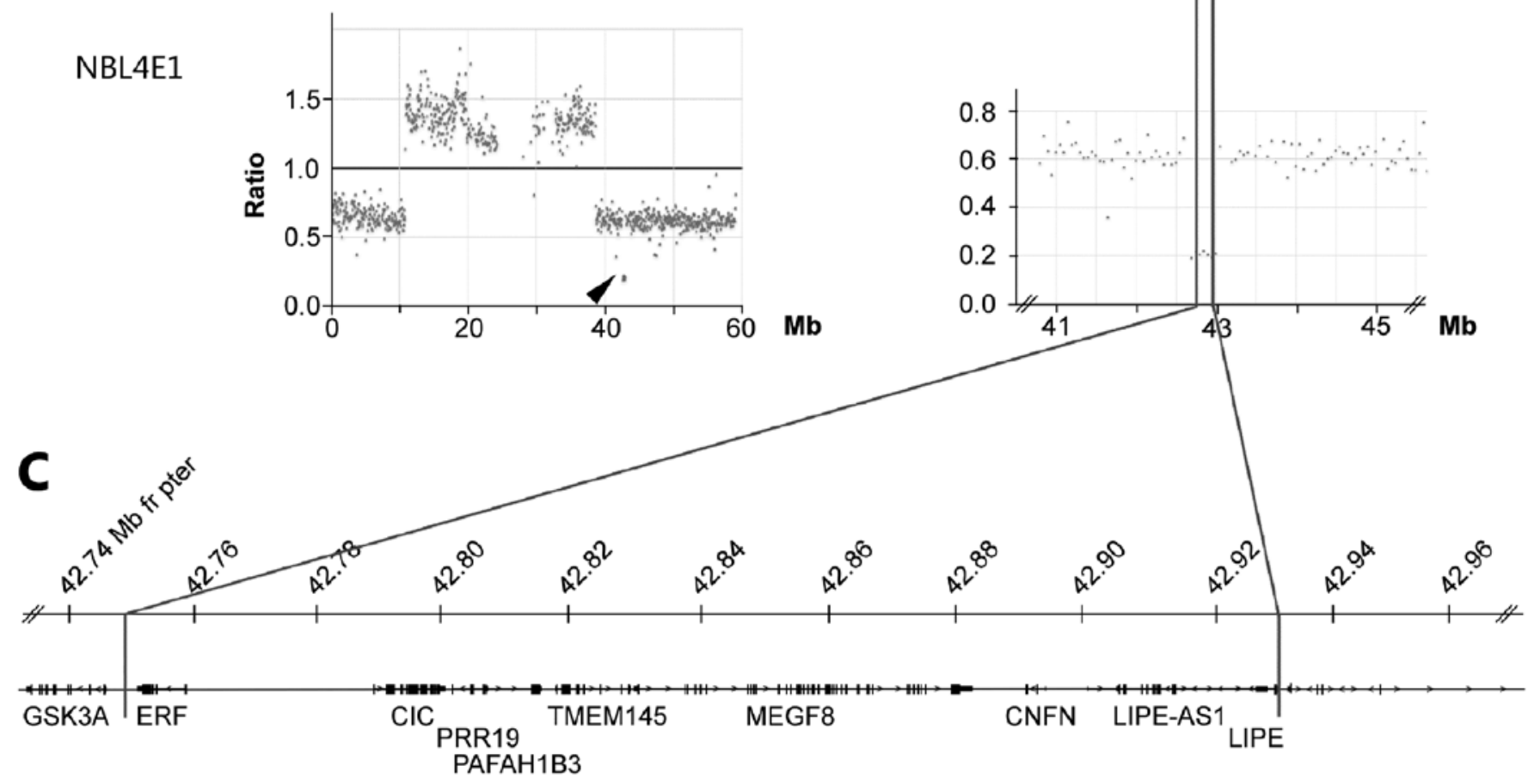

B

Chr19

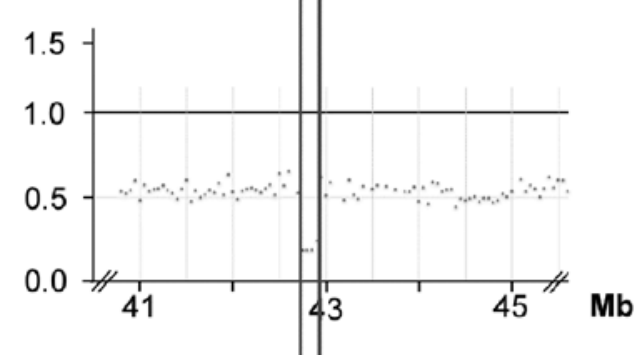

Figure 3. Deletions at chromosomal region $19 \mathrm{q} 13.2$ causes homozygous loss of CIC. (A) Three tumors with larger deletions of $19 \mathrm{q}$ also display smaller focal deletions causing homozygous loss at 19q13.2 as indicated by black arrows. (B) The shortest region of overlapping deletions are delimitated by sample NBL13E6 at position 42.75-42.93 Mb from pter end and (C) includes the genes ERF, CIC, PRR19, PAFAH183, TMEM145, MEGF8, CNFN, LIPE-AS1 and LIPE.

zygous deletion was also observed distally the CIC locus at 43.34-43.53 Mb from pter in sample NBL12E3. However, this particular region contains several pregnancy-specific glycoprotein (PSG) genes that previously have been shown to inhabit various copy number polymorphisms.

Besides identifying genomic copy number alterations we could detect tumor specific junctions in $86 \%$ of the
$M Y C N$-amplified tumors in using the boundaries from offtarget reads in amplified regions. As these junctions are likely to be highly specific for each tumor, and by definition not present in normal DNA, they could be used to monitor disease through analysis of circulating tumor-DNA.

Collectively, we show that copy number profiles generated from normalized coverage of exome sequencing are easily 
interpreted through the web based Shiny application with similar resolution as the Affymetrix 250K SNP-arrays. The extended use of exome sequencing beyond variant- and indel calling is of particular interest in neuroblastoma tumor biology as genomic profiling is of uttermost importance in the clinical evaluation of these tumors. Use of exome sequencing has the advantage compared to other methods for copy number variants, in that it can also identify protein changing events that can be used for gene targeted therapy such as the ALK specific inhibitor Crizotinib.

\section{Acknowledgements}

The authors wish to thank the Genomics and Bioinformatics Core Facility platforms at Sahlgrenska Academy, University of Gothenburg, Gothenburg, Sweden for access and assistance with instrumentation and analysis. This study was supported with grants from the Swedish Childhood Cancer Foundation (S.F.; NBCNSPDHEL10/021, NCp2015-0061, TJ2014-0064. T.M.; PR2013-0102), the Swedish Cancer Foundation (T.M.; 14-0342), The Swedish state under the LUA/ALF agreement (ALFGBG-447171), Project grant from Laboratory division Sahlgrenska University Hospital, Lions Cancerfond Väst, The Selma Anderson Foundation, Fondkistan, Assar Gabrielssons Foundation, Sahlgrenska University Hospital Foundations, the Swedish Research Council (T.M./S.F.; 2014-3031), the Swedish Foundation for Strategic Research (T.M.; RB13-0204).

\section{References}

1. Cohn SL, Pearson AD, London WB, Monclair T, Ambros PF Brodeur GM, Faldum A, Hero B, Iehara T, Machin D, et al; INRG Task Force: The International Neuroblastoma Risk Group (INRG) classification system: An INRG Task Force report. J Clin Oncol 27: 289-297, 2009.

2. Carén H, Kryh H, Nethander M, Sjöberg RM, Träger C, Nilsson S, Abrahamsson J, Kogner P and Martinsson T: High-risk neuroblastoma tumors with 11q-deletion display a poor prognostic chromosome instability phenotype with later onset. Proc Natl Acad Sci USA 107: 4323-4328, 2010.

3. Janoueix-Lerosey I, Schleiermacher G, Michels E, Mosseri V, Ribeiro A, Lequin D, Vermeulen J, Couturier J, Peuchmaur M, Valent A, et al: Overall genomic pattern is a predictor of outcome in neuroblastoma. J Clin Oncol 27: 1026-1033, 2009.

4. Cetinkaya C, Martinsson T, Sandgren J, Träger C, Kogner P, Dumanski J, Díaz de Ståhl T and Hedborg F: Age dependence of tumor genetics in unfavorable neuroblastoma: arrayCGH profiles of 34 consecutive cases, using a Swedish 25-year neuroblastoma cohort for validation. BMC Cancer 13: 231, 2013.

5. Combaret V, Iacono I, Bréjon S, Schleiermacher G, Pierron G, Couturier J, Bergeron C and Blay JY: Analysis of genomic alterations in neuroblastoma by multiplex ligation-dependent probe amplification and array comparative genomic hybridization: A comparison of results. Cancer Genet 205: 657-664, 2012.

6. Ambros IM, Brunner B, Aigner G, Bedwell C, Beiske K, Bénard J, Bown N, Combaret V, Couturier J, Defferrari R, et al: A multilocus technique for risk evaluation of patients with neuroblastoma. Clin Cancer Res 17: 792-804, 2011.
7. Sathirapongsasuti JF, Lee H, Horst BA, Brunner G, Cochran AJ, Binder S, Quackenbush J and Nelson SF: Exome sequencingbased copy-number variation and loss of heterozygosity detection: Exome CNV. Bioinformatics 27: 2648-2654, 2011.

8. Krumm N, Sudmant PH, Ko A, O'Roak BJ, Malig M, Coe BP, Quinlan AR, Nickerson DA and Eichler EE; NHLBI Exome Sequencing Project: Copy number variation detection and genotyping from exome sequence data. Genome Res 22: 1525-1532, 2012.

9. Valdés-Mas R, Bea S, Puente DA, López-Otín C and Puente XS: Estimation of copy number alterations from exome sequencing data. PLoS One 7: e51422, 2012.

10. Boeva V, Popova T, Bleakley K, Chiche P, Cappo J, Schleiermacher G, Janoueix-Lerosey I, Delattre $\mathrm{O}$ and Barillot E: Control-FREEC: A tool for assessing copy number and allelic content using next-generation sequencing data. Bioinformatics 28: 423-425, 2012.

11. Carén H, Erichsen J, Olsson L, Enerbäck C, Sjöberg RM, Abrahamsson J, Kogner P and Martinsson T: High-resolution array copy number analyses for detection of deletion, gain, amplification and copy-neutral LOH in primary neuroblastoma tumors: Four cases of homozygous deletions of the CDKN2A gene. BMC Genomics 9: 353, 2008.

12. Chang WCJ, Allaire JJ, Xie Y and McPehrson J: Shiny: Web Application Framework for R. R package version 0.12.0. R package version 0120 2015. https://cran.r-project.org/web/ packages/shiny/index.html. Accessed August 5, 2015.

13. Hoefling H: A Path Algorithm for the Fused Lasso Signal Approximator. J Comput Graph Stat 19: 984-1006, 2010.

14. Molenaar JJ, Koster J, Zwijnenburg DA, van Sluis P, Valentijn LJ, van der Ploeg I, Hamdi M, van Nes J, Westerman BA, van Arkel J, et al: Sequencing of neuroblastoma identifies chromothripsis and defects in neuritogenesis genes. Nature 483: 589-593, 2012.

15. Pandey GK, Mitra S, Subhash S, Hertwig F, Kanduri M, Mishra K, Fransson S, Ganeshram A, Mondal T, Bandaru S, et al: The risk-associated long noncoding RNA NBAT-1 controls neuroblastoma progression by regulating cell proliferation and neuronal differentiation. Cancer Cell 26: 722-737, 2014.

16. Pugh TJ, Morozova O, Attiyeh EF, Asgharzadeh S, Wei JS, Auclair D, Carter SL, Cibulskis K, Hanna M, Kiezun A, et al: The genetic landscape of high-risk neuroblastoma. Nat Genet 45: 279-284, 2013

17. Sausen M, Leary RJ, Jones S, Wu J, Reynolds CP, Liu X Blackford A, Parmigiani G, Diaz LA Jr, Papadopoulos N, et al: Integrated genomic analyses identify ARID1A and ARID1B alterations in the childhood cancer neuroblastoma. Nat Genet 45: 12-17, 2013.

18. Boeva V, Zinovyev A, Bleakley K, Vert JP, Janoueix-Lerosey I, Delattre $\mathrm{O}$ and Barillot E: Control-free calling of copy number alterations in deep-sequencing data using GC-content normalization. Bioinformatics 27: 268-269, 2011.

19. Jiménez G, Shvartsman SY and Paroush Z: The Capicua repressor - a general sensor of RTK signaling in development and disease. J Cell Sci 125: 1383-1391, 2012.

20. Eisenreich S, Abou-El-Ardat K, Szafranski K, Campos Valenzuela JA, Rump A, Nigro JM, Bjerkvig R, Gerlach EM, Hackmann K, Schröck E, et al: Novel CIC point mutations and an exon-spanning, homozygous deletion identified in oligodendroglial tumors by a comprehensive genomic approach including transcriptome sequencing. PLoS One 8: e76623, 2013.

21. Sjöblom T, Jones S, Wood LD, Parsons DW, Lin J, Barber TD, Mandelker D, Leary RJ, Ptak J, Silliman N, et al: The consensus coding sequences of human breast and colorectal cancers. Science 314: 268-274, 2006.

22. Choi N, Park J, Lee JS, Yoe J, Park GY, Kim E, Jeon H, Cho YM, Roh TY and Lee Y: miR-93/miR-106b/miR-375-CICCRABP1: A novel regulatory axis in prostate cancer progression. Oncotarget 6: 23533-23547, 2015. 